\title{
Multifocal plasmacytoma presenting with upper gastrointestinal bleeding, chronic diarrhea, and multiple sites of small-bowel intussusception
}

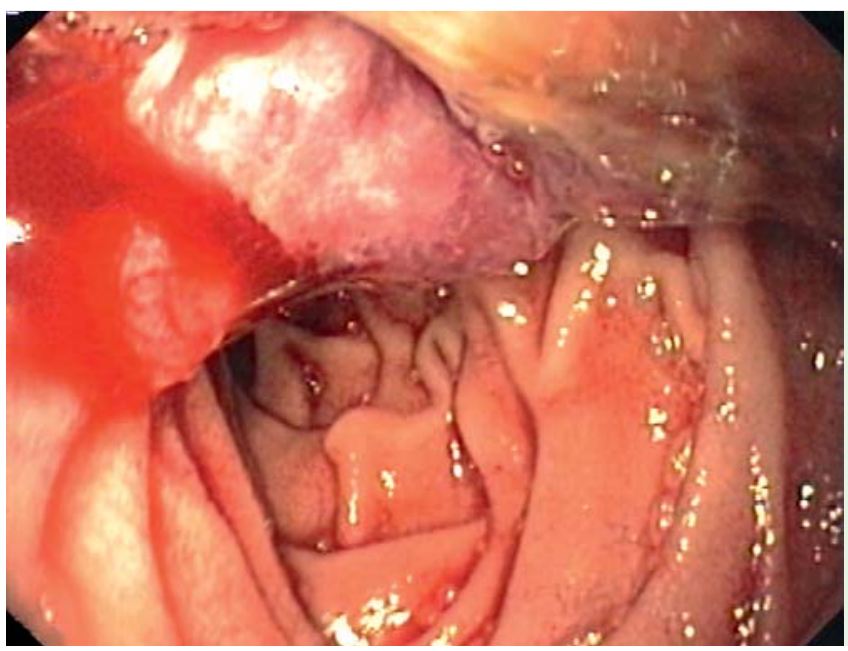

Fig. 1 Endoscopic view of a duodenal mass in a 48-year-old with intermittent abdominal pain, chronic diarrhea, and acute massive upper gastrointestinal bleeding.

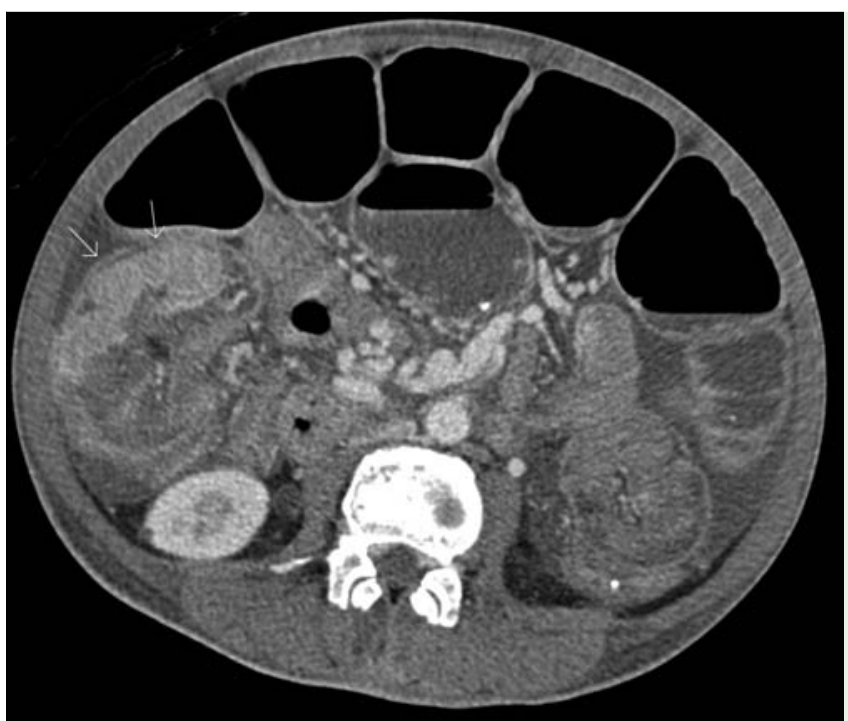

Fig. 3 Computed tomography (CT) showing the two sites of small-bowel intussusception.

A 48-year-old man was referred to our hospital because of intermittent abdominal pain and chronic diarrhea for 2 months and acute massive upper gastrointestinal bleeding, which had commenced a few days ago. Esophagogastroduodenoscopy (EGD) showed an ulcerating mass in the second part of the duodenum, located just beyond the ampulla ( $\bullet$ Fig. 1 ). The bleeding was successfully stopped by injecting epinephrine into the tumor followed by spraying with Histoacryl [1] ( $\bullet$ Fig.2). A few days after the procedure the patient developed acute, severe abdominal pain and nausea and vomiting. Computed tomography (CT) showed intussusception of two long segments of the ileum associated with multiple intraluminal masses. The first segment was in the jejunum and the second was ileocolic intussusception ( $\bullet$ Fig.3). There were also multiple bony metastases and lymphadenopathy in the paravertebral region. A biopsy sample from the duodenum showed hematological malignancy with plasmacytic differentiation, and was positive for CD45, CD138, c-lambda, and c-kappa, and negative for CD20, CD30, and CD3 ( Fig. 4). The bone

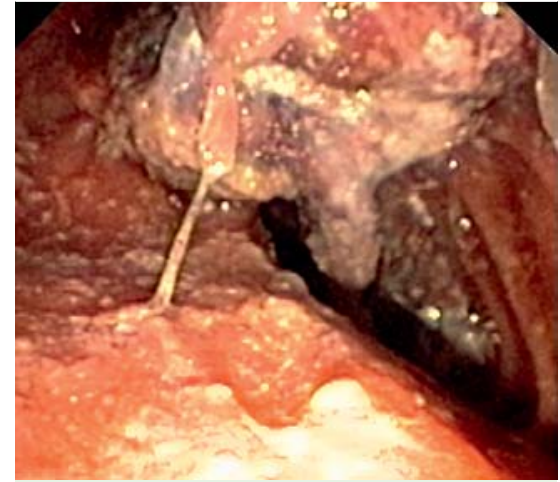

Fig. 2 Bleeding stopped after spraying Histoacryl.

marrow biopsy was compatible with multiple myeloma. After treatment with intravenous chemotherapy, the patient's clinical condition improved and the abdominal pain as well as small-bowel obstruction disappeared a few days later.

Abnormalities in B-cell transformation result in the development of an aberrant or malignant plasma cell called the plasmablast. These cells can proliferate in the bone marrow, giving rise to multiple myeloma or primary extramedullary plasmacytoma, which is a rare form of the disease [2]. Extramedullary plasmacytoma accounts for $20 \%$ of plasmablast-related diseases but only $7 \%$ of cases involve the gastrointestinal tract. The most commonly involved organs, in the order of decreasing frequency, are the stomach, jejunum, ileum, colon, and rectum [3]. Duodenal involvement is rare [2-5]. Endoscopic findings in plasmacytoma show no specific characteristics: it may manifest as discrete ulcers, ulcerating mass, thickening of the mucosal fold, or even as a mucosal polyp [2]. The differential diagnoses are duodenal lymphoma, adenocarcinoma of the duodenum, and pancreatic head cancer invading the duodenum. Hence the final diagnosis is based on the histopathology and immunohistochemistry.

Endoscopy_UCTN_Code_CCL_1AB_2AZ_3AB

\section{Competing interests: None}




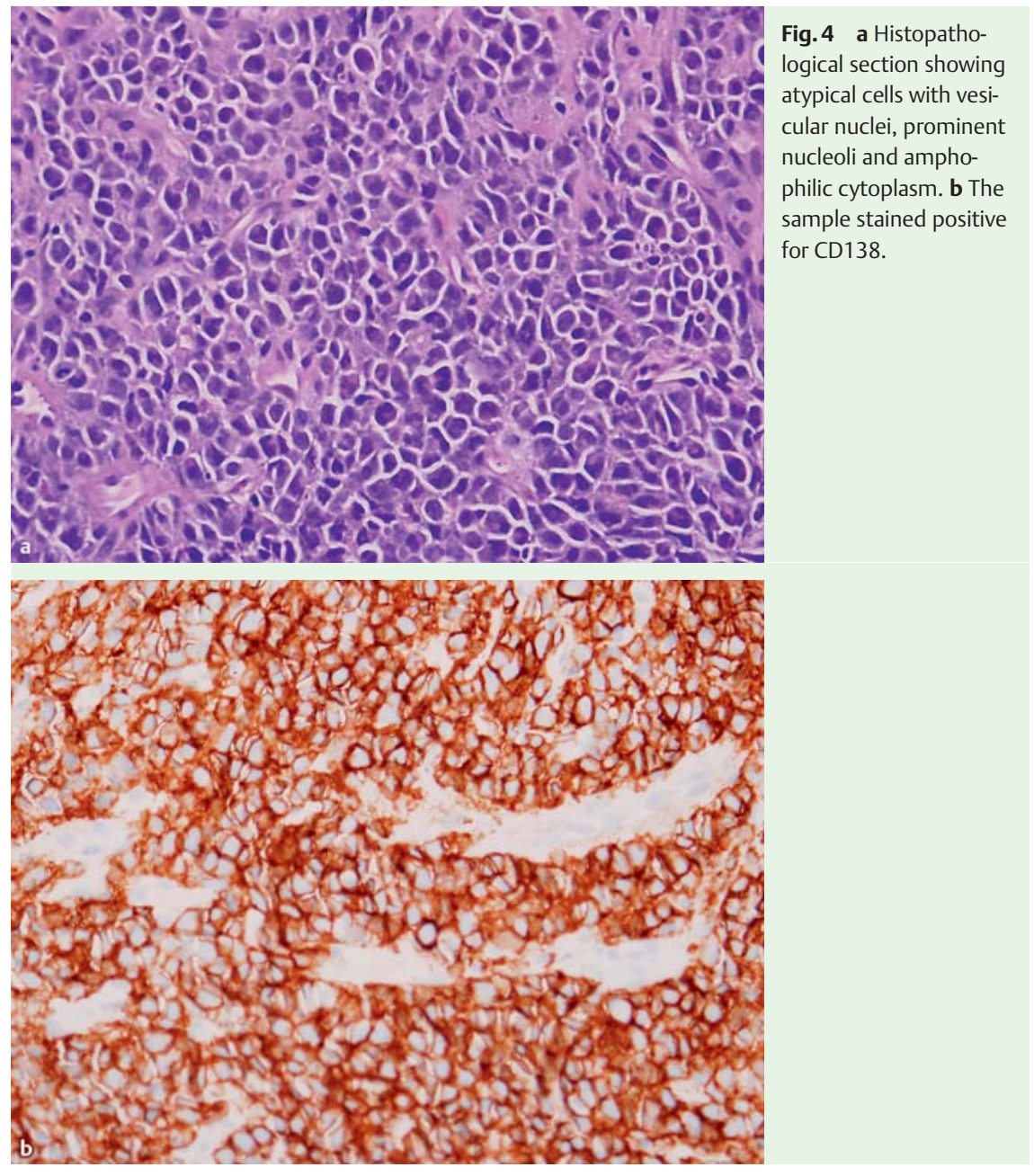

\section{Prachayakul' ${ }^{1}$ P. Aswakul ${ }^{2}$, M. Deesomsak ${ }^{2}$, A. Pongpaibul ${ }^{3}$}

${ }^{1}$ Siriraj Gl Endoscopy Center, Division of Gastroenterology, Department of Internal Medicine, Faculty of Medicine, Siriraj Hospital, Mahidol University, Bangkok, Thailand

${ }^{2}$ Liver and Digestive Institute, Samitivej Sukhumvit Hospital, Bangkok, Thailand

${ }^{3}$ Department of Pathology, Faculty of Medicine, Siriraj Hospital, Mahidol University, Bangkok, Thailand

\section{References}

1 Prachayakul V, Aswakul P, Kachinthorn U. Spraying N-butyl-2-cyanoacrylate (Histoacryl) as a rescue therapy for gastrointestinal malignant tumor bleeding after failed conventional therapy. Endoscopy 2011; 43: E227-228

2 Karam AR, Semaan RJ, Buch $K$ et al. Extramedullary duodenal plasmacytoma presenting with gastric outlet obstruction and painless jaundice. Radiol Cases 2010; 4: $22-28$

3 Kim MH, Lee SK, Seo DW et al. Tumor of the major duodenal papilla. Gastrointest Endosc 2001; 54: 609-620

4 Chua SC, O'Connor SR, Wong WL et al. Solitary plasmacytoma of bone with oncogenic osteomalacia: recurrence of tumour confirmed by PET/CT. A case report with a review of the radiologist literature. $\mathrm{Br} \mathrm{J}$ Radiol 2008; 81: e110-114

5 Athanasopoulos P, Arkadopoulos N, Stafyla V et al. A rare combination of an endocrine tumour of the common bile duct and a follicular lymphoma of the ampulla of Vater: a case report and review of the literature. World J Surg Oncol 2011; 9: 4-7

Bibliography

DOI http://dx.doi.org/

10.1055/s-0032-1326262

Endoscopy 2013; 45: E84-E85

(c) Georg Thieme Verlag KG

Stuttgart · New York

ISSN 0013-726X

\section{Corresponding author \\ V. Prachayakul}

Division of Gastroenterology

Department of Internal Medicine,

Faculty of Medicine

Siriraj Hospital

Mahidol University

Bangkok

Thailand 10700

Fax: +662-4196101

kaiyjr@gmail.com 